\title{
Spreading Model for Wall Films Generated by High-Pressure Sprays
}

\author{
Q. Lamiel ${ }^{1,2}, \mathrm{~N}$. Lamarque ${ }^{1,{ }^{*}}, \mathrm{~J}$ Hélie $^{1}, \mathrm{D}$. Legendre ${ }^{2}$ \\ ${ }^{1}$ Continental Automotive SAS, 1 avenue Paul Ourliac BP 1149, 31036 Toulouse, France \\ ${ }^{2}$ Institut de Mécanique des Fluides de Toulouse (IMFT) - Université de Toulouse, \\ CNRS-INPT-UPS, Toulouse FRANCE \\ ${ }^{*}$ Corresponding author: nicolas.lamarque@continental-automotive.com
}

\begin{abstract}
This paper presents a new model developed to predict the area of wall films that may develop in gasoline direct injection engines (GDI). In a always more restrictive legislation on gas emissions the injection process in internal combustion (IC) engines has been highlighted as a domain of great concern in order to satisfy these requirements. Many spray wall interactions models exist in literature and are included in different CFD tools. Most often they are based on the sum of single drop-wall impacts. The specificity of the present model lies in its simplicity and the way the film is treated globally. Here its propagation is predicted using a balance between the momentum given by the spray and the viscous shear stress. Jointly with the theoretical model, an experimental set-up has been built up, an optical measurement technique called Refractive Index Matching method is used to follow the development of the wall film.

It has been found that the area of the wall film is proportional to the duration of injection, while the distance between the injector and the wall has not shown many influence on the evolution of area. The influence of the injection pressure has also been identified, when the pressure is doubled the radius of the film is multiplied by $\sqrt[3]{2}$. Eventually the model predicts that film thickness decreases as fuel pressure rises.
\end{abstract}

\section{Keywords}

automotive sprays, spray-wall impingement, refractive index matching method

\section{Introduction}

Fuel impingement in an internal combustion (IC) engine is a critical phenomenon. Despite progresses in gasoline direct injection (GDI) sytem design, fuel spray-wall impingement still happens for certain engine conditions and leads to liquid deposits. These wall films on the piston or the cylinder liner strongly affect mixture formation and entail bad local mixture homogeneity. This has been identified as a major source of pollutant emissions, especially particulate matter $[1,2]$. As a consequence, it is essential to have a good understanding of these spray-wall interactions and identify the key factors to keep them under control.

Drop-wall impingement is a topic widely studied in literature, because many applications (automotive, aeronautics, cooling, fire safety, printing, painting, agrochemicals, phamaceutical to name some) strongly depend on the performances of atomisers and spray systems [3]. Many models have been derived with success to describe the different impact regimes (on cold or hot surfaces) and the spreading characteristics of single droplets $[4,5,6]$. Some of them are commonly used in CFD tools and coupled with a Lagrangian description of the spray [7, 8, 9].

Nevertheless, it has been shown that spray-wall impingement implies many phenomena, which cannot be completly described by single droplet-wall impact models $[5,10]$. Moreover, while available computational power has strongly increased and despite some success, CFD tools sometimes remain expensive to simulate all the different engine conditions, for a first screening or even while optimising injector targeting and injection timings. In the present paper, a simple model based on basic conservation laws is proposed to describe wall film spreading in non- or low-volatile conditions. The global approach used to develop this model makes its originality, as well as the fact that it focuses on the film, rather than the spray dynamics. It is compared and validated with experimental measurements using the refractive index matching method and high-speed video imaging.

\section{Spray-wall interactions and experimental setup}

When a GDI spray impinges upon the wall, the very numerous liquid droplets $\left(10^{9}\right.$ to $\left.10^{10}\right)$ are either deposited or splashed and pushed away because of the high momentum, as depicted on Figure 1. In non-vaporising conditions, the deposited liquid mass quickly generates a continuous wall film. It then propagates under the effect of mass deposition and the spray dynamic pressure. While both the spray and the film can be observed on classical shadowgraph pictures like Figure 1, the film spreading can be better tracked with the refractive index matching (RIM) method.

The experimental setup is presented in Figure 3. It is here composed of a GDI injector (b), fed by a high-pressure pump (a), which generates a spray (c) that impinges upon a transparent roughened quartz plate (e); the impingement and the spreading of the liquid are recorded with a high speed video camera $(\mathrm{g})$. The camera is directed towards a mirror ( $f$ ) placed under the impingement plate in order to observe the phenomena from below. The light source (d) is an optical fiber which illuminates the quartz plate at grazing angle. This method of visualisation is 


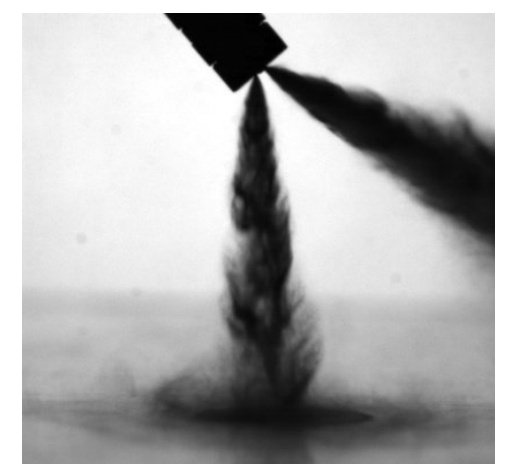

Figure 1. GDI spray impinging upon a smooth plate. Fuel pressure is $100 \mathrm{bar}$.

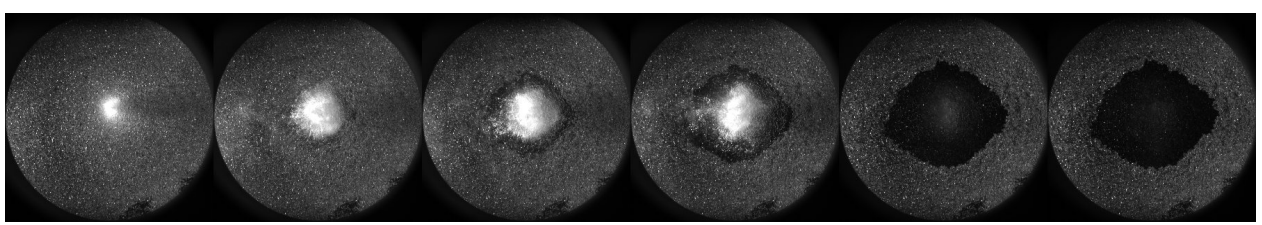

Figure 2. Images of injection as visualised on the RIM setup for $P_{i}=200 \mathrm{bar} T_{i}=5 \mathrm{~ms}$ and $d_{W}=50 \mathrm{~mm}$ images are displayed every $1.5 \mathrm{~ms}$

called Refractive Index Matching method (RIM) and it has originally been introduced by Drake [1] to study a DI engine then used by Yang and Gandhi in a Diesel engine [11]. Finally Maligne and Bruneaux [12] used it in a pressure chamber to compare wall films created by different types of GDI injectors. As the liquid fills in the surface troughs, the illuminated plate scatters less light towards the camera. This enables to clearly identify the wall film footprint and its surface can be evaluated (see Figure 2). After a fine calibration, the wall film thickness is quantified $[1,11,12]$. For the liquid films present in this study the accuracy is $\pm 1 \mu \mathrm{m}$. The injector used for this study has three holes. In this particular case, it is tilted by $30^{\circ}$, hence one of the plumes impacts upon the plate vertically and the spreading is homogeneous in the radial direction. Using a specially developed image post-processing, the edge of the liquid film can be tracked through time. Considering the camera resolution $(1 \mathrm{px}=0.17 \mathrm{~mm})$ the accuracy on the film radius is $1 \mathrm{~mm}$ close to the impinging zone and it becomes better and better as the film is spreading.

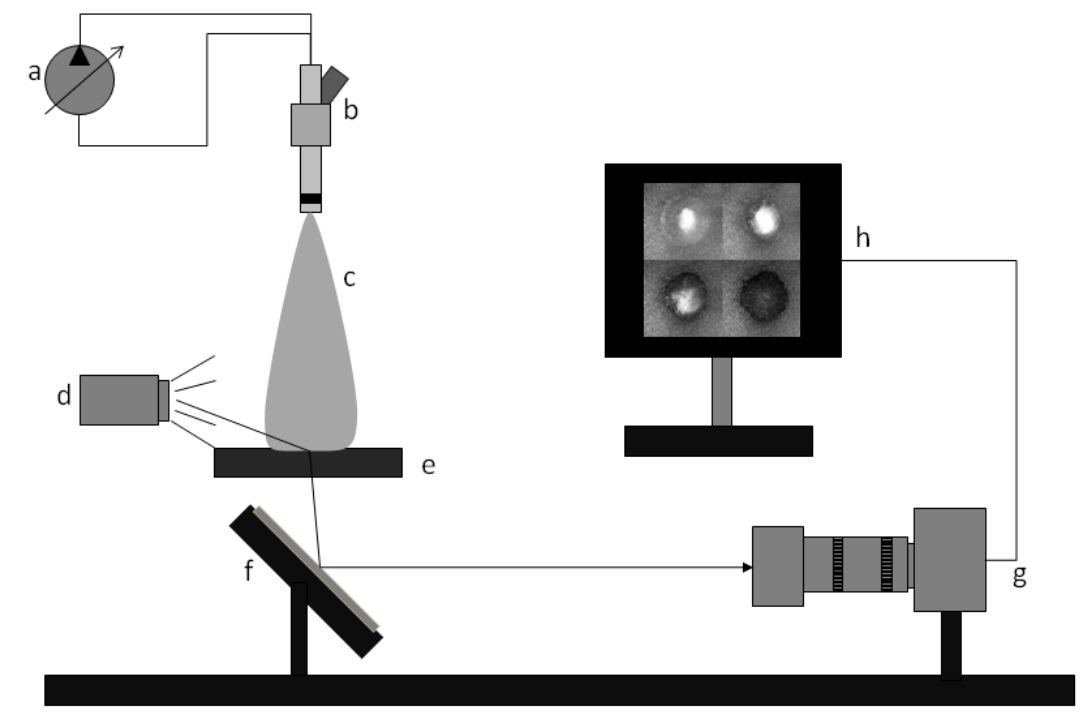

Figure 3. Sketch of the Refractive Index Matching experimental setup 


\section{Wall-film spreading modelling}

As very well underlined in [10], spray-wall impingement is a very complex phenomenon with many interactions between the incoming droplets and the perturbated film surface. Taking into account all the details (especially because the film is strongly perturbated by many simultaneous impacts) would be a tremendous effort. The purpose here is not to develop yet another very fine drop-wall interaction model to be implemented in a CFD tool. In this section is derived a simple global model, which describes the wall film propagation. The first hypothesis is the wall film has a puddle or a pizza shape, with a homogeneous thickness $e$ (see Figure 4). Surface tension effects, which are strong at the wall film edge and create a rim, are here neglected. Only a steady-state balance between a driving (spray pressure / momentum transfer) and a counteracting force in the radial propagation direction is considered.

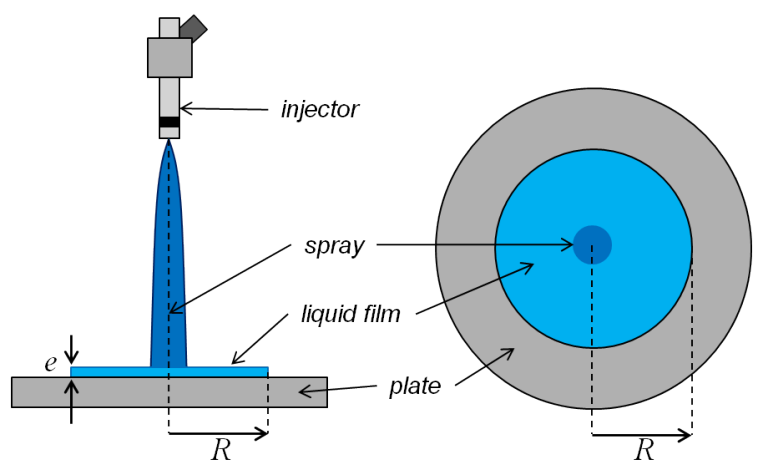

Figure 4. Sketch of the modelled problem. The film thickness is supposed to be homogeneous.

The wall film spreading model relies on the use of the mass and momentum conservation equations, outside the impact zone. Considering the characteristic lengths and times (thickness $e \simeq 10 \mu \mathrm{m}$, radius $R \simeq 10 \mathrm{~mm}$, injection time $T_{i} \simeq 1 \mathrm{~ms}$ ), the thin wall film is supposed to be in the lubrication regime. Considering that the mass is totally $\left(K_{m}=1\right)$ or partially $\left(K_{m} \leq 1\right)$ transferred in the film, the mass balance writes:

$$
\frac{d \pi R^{2} e}{d t}=K_{m} Q
$$

Where $Q$ stands for the injection discharge of the injector, then assuming a constant film thickness and that $Q$ is constant during the injection process:

$$
R=\sqrt{\frac{K_{m} Q t}{\pi e}}
$$

This shows that the radius of the film is proportional to the square root of time, the thickness of the film needs to be derivated to exactly know the evolution of the film.

Now looking at the momentum balance and keeping in mind that the film is in the lubrication regime (i.e. the flow is then parallel and pressure only depends on $r$ ):

$$
\frac{\partial p}{\partial r}=\eta \frac{\partial^{2} v_{r}}{\partial z^{2}}
$$

On the one hand a simple approximation on the pressure gradient gives:

$$
\frac{\partial p}{\partial r} \approx \frac{\Delta P}{R} .
$$

Defining $\Delta P=P_{s}-P_{a}$ and $P_{s}=P_{a}+P_{d}$ then $\Delta P=P_{d}$, which is the dynamic pressure given by the spray or, introducing $K_{p}$ to take into account the fact that the film pressure in the impingement zone may not be equal to the spray total pressure $\Delta P=K_{P} P_{d}$.

On the other hand stress can be approximated as:

$$
\eta \frac{\partial^{2} v_{r}}{\partial z^{2}} \approx \frac{\eta V}{e^{2}}
$$

where $V$ is the depth-averaged radial velocity. It can be used to express the wall film spreading velocity, by writing $V=\dot{R}$. Expressing $e$ with the help of Eq 3 - 5 we get:

$$
e=\sqrt{\frac{\eta V R}{2 \pi K_{P} P_{d}}}
$$


and with $V R \propto Q / e$ we get an expression for the thickness:

$$
e \propto \sqrt[3]{\frac{\eta Q}{P_{d}}}
$$

As the discharge coefficient $Q$ is varying like $P_{i}^{1 / 2}$, a trend for the evolution of $e$ and $R$ is found:

$$
e \propto P_{i}^{-1 / 6} \text { and } R \propto P_{i}^{1 / 3}
$$

For convenience Eq $2 \& 7$ can be rewritten using $K_{P}, K_{m}, \eta, P_{d}$ and $Q$ and it comes:

$$
\begin{aligned}
& e=\sqrt[3]{\frac{\eta K_{m} Q}{2 \pi K_{P} P_{d}}} \\
& R^{6}-R_{0}^{6}=K \frac{P_{d} Q^{2}}{\pi^{2} \eta} t^{3},
\end{aligned}
$$

where $R_{0}$ stands for the impinging zone of the spray, inside this zone the flow is more complex and the accuracy of the model is not guaranteed. And the prefactor $K$ is defined as :

$$
K=2 K_{P} K_{m}^{2},
$$

and takes into account that the spray mass and momentum may only be partially transferred to the liquid film. In what follows, we set $K_{P}=1$ for simplicity, which means that the pressure at the film centre is the spray dynamic pressure. The value of $K_{m}$ will be discussed in the next section.

\section{Results and discussion}

This present paragraph shows the results obtained applying the RIM method and the comparison with the proposed model. Table 1 summarises the different operating points. Fuel pressure $P_{i}$, injection time $T_{i}$ and injector-wall distance $d_{W}$ have been varied within realistic ranges with respect to automotive applications.

Table 1. Operating points tested in the present study

\begin{tabular}{c|cc}
\hline Factor & Unit & Values \\
\hline Fuel pressure $P_{i}$ & bar & $50,100,200$ \\
Injection time $T_{i}$ & $\mathrm{~ms}$ & 1 to 5 \\
Injector-wall distance $d_{W}$ & $\mathrm{~mm}$ & $30,40,50,60,70$
\end{tabular}

Figure 5 shows how the wall film area evolves in time. Whatever the injection time, the area growths collapse and, in the steady-state range are proportional to time. Here, only $d_{W}=50 \mathrm{~mm}$ is displayed, as the injector wall-distance has very little influence on the phenomenon. Hence, it confirms the radius evolution as the square root of time, as shown by Eq 2. It can also be observed that, once the injection is finished, the wall film still has some inertia and continues spreading at a much slower rate; the assumption of a constant thickness is then questionable.

Figure 6 shows the film thickness obtained with the calibration of the RIM method. The gray level is roughly homogeneous on the whole puddle and variations are mainly due to the surface irregularities that can scatter light differently, the profile is made $2 \mathrm{~s}$ after the start of injection. A steady state is reached and it is assumed that, no droplets that could disturb the measure, are present above the film surface. Hence a measurement of the mean film thickness is realised knowing the film surface. A value of $3.7 \mu \mathrm{m}$ is found, with the assumption of constant thickness we can calculate the deposited mass of heptane. It is compared to the injected mass, and it provides the mass ratio $K_{m}=15.11 \%$

A reasonable assumption is to say that a part of the mass deposited at the end of injection has already vaporised after $2 \mathrm{~s}$. After a quick evaluation based on experiments about vaporisation rates of heptane droplets gently deposited on a plate, and the use of a 1D model that predicts lifetime of liquid films [13], we can say that almost twice the mass was present at the end of injection. This gives a new value for $K_{m}=29 \%$, which is in line with results previously obtained in the literature $[11,14,15,16]$. Then, the thickness at the end of injection is obtained using Eq 12:

$$
e_{\left(t=T_{i}\right)}=\frac{e_{(t=2 \mathrm{~s})} A_{(t=2 \mathrm{~s})}}{A_{\left(t=T_{i}\right)}}
$$

The thickness of the film at the end of injection is $14.8 \mu \mathrm{m}$.

On the other hand the model gives with Eq 9 and the slope of Figure 5 a thickness of $16.8 \mu \mathrm{m}$ that is to say a relative error of $12 \%$ of the measured thickness. 


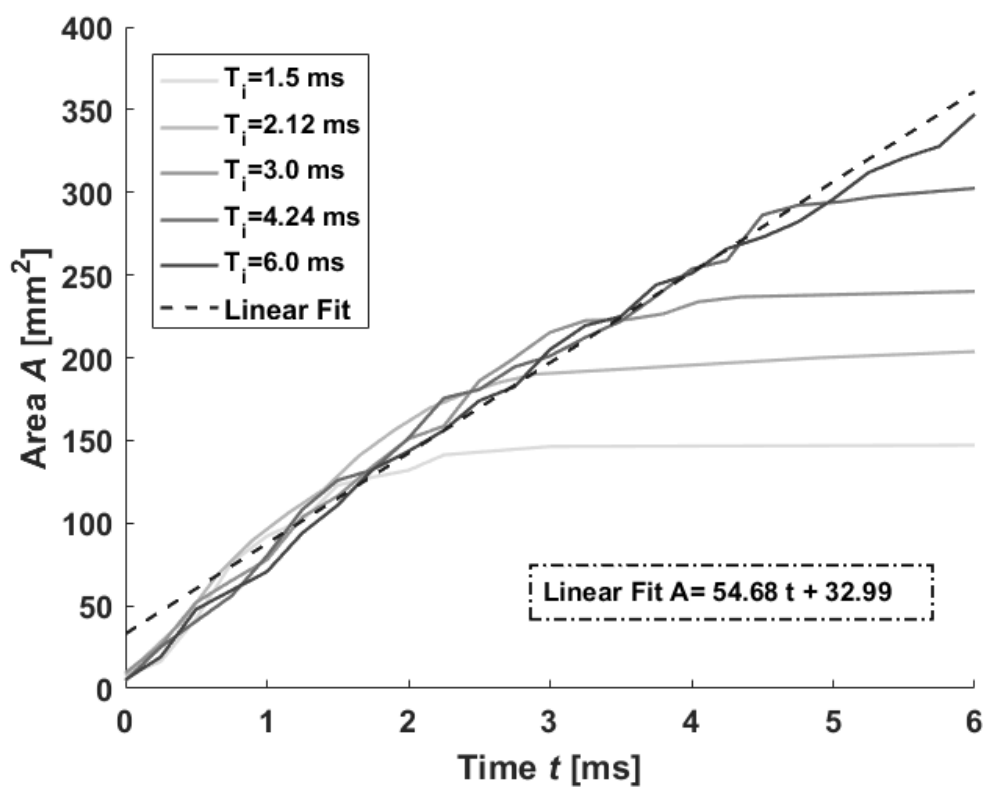

Figure 5. Wall film area time evolution for the operating point $P_{i}=100 \mathrm{bar}, d_{W}=50 \mathrm{~mm}$ and different injection times.

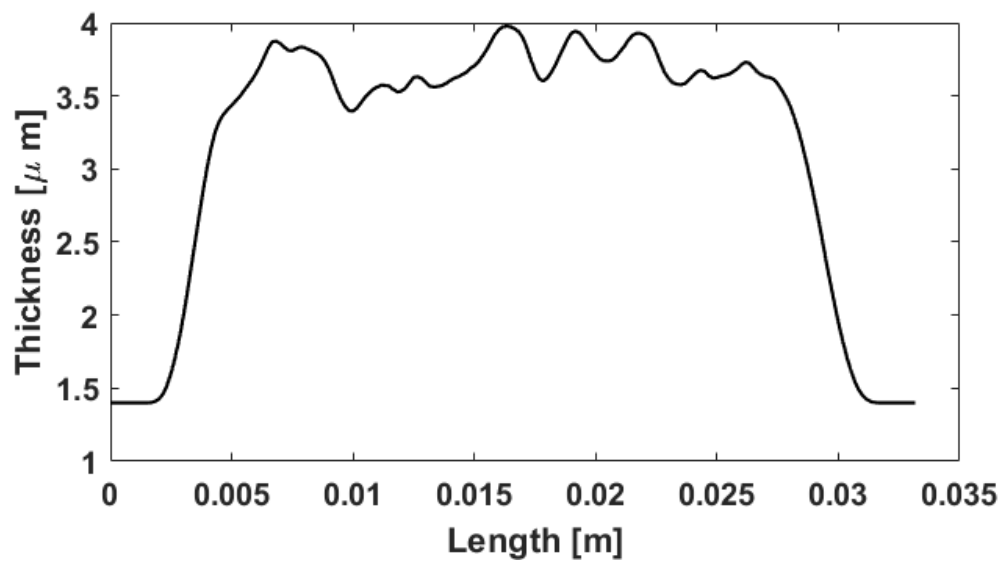

Figure 6. Thickness profile for a film of heptane obtained $2 \mathrm{~s}$ after the end of injection; $P_{i}=100 \mathrm{bar}, d_{W}=60 \mathrm{~mm}, T_{i}=5.0 \mathrm{~ms}$

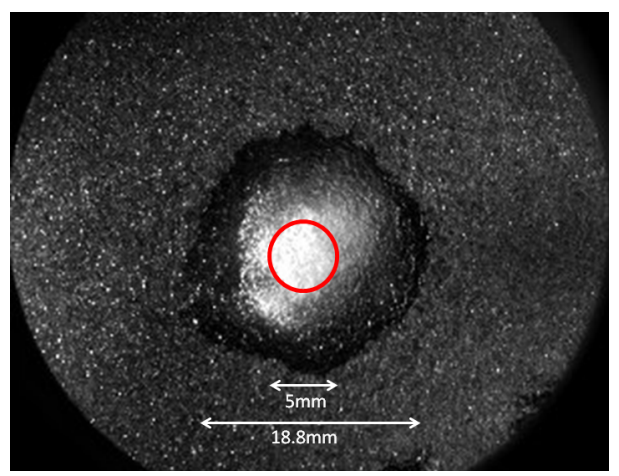

Figure 7. Normal impingement of spray on rough plate; $P_{i}=100 \mathrm{bar} d_{W}=60 \mathrm{~mm} T_{i}=5.0 \mathrm{~ms}$. Red zone highlights the area where momentum is transferred to the film

It has been shown in previous studies that there is less deposited mass when fuel pressure increases [14]. Nevertheless, from 50 to 200bar, changes of $K$ are not expected to be very high. Then, for simplicity, $K_{m}$ is set to 0.29 in what follows which gives a value of 0.58 for $K$. According to Figure 5 and to Eq 10 a value of $12.5 \times 10^{3} \mathrm{~Pa}$ for $P_{d}$ is obtained. To understand the meaning of this value a simple calculation is performed, taking a value of $K_{P}=1$ 
(which is what we assume in the model) means that the momentum is conserved from the injector hole to the impact plate. In other words the pressure force is constant, which can be expressed by:

$$
P_{d} A_{s}=P_{i} A_{o}
$$

where $A_{s}$ is the area where the spray momentum is transferred to the liquid film and $A_{o}$ is the area of the injector hole, then $A_{s}=18.2 \mathrm{~mm}^{2}$ which correspond to a radius of $2.4 \mathrm{~mm}$ which is really close to what we can observe on Figure 7.

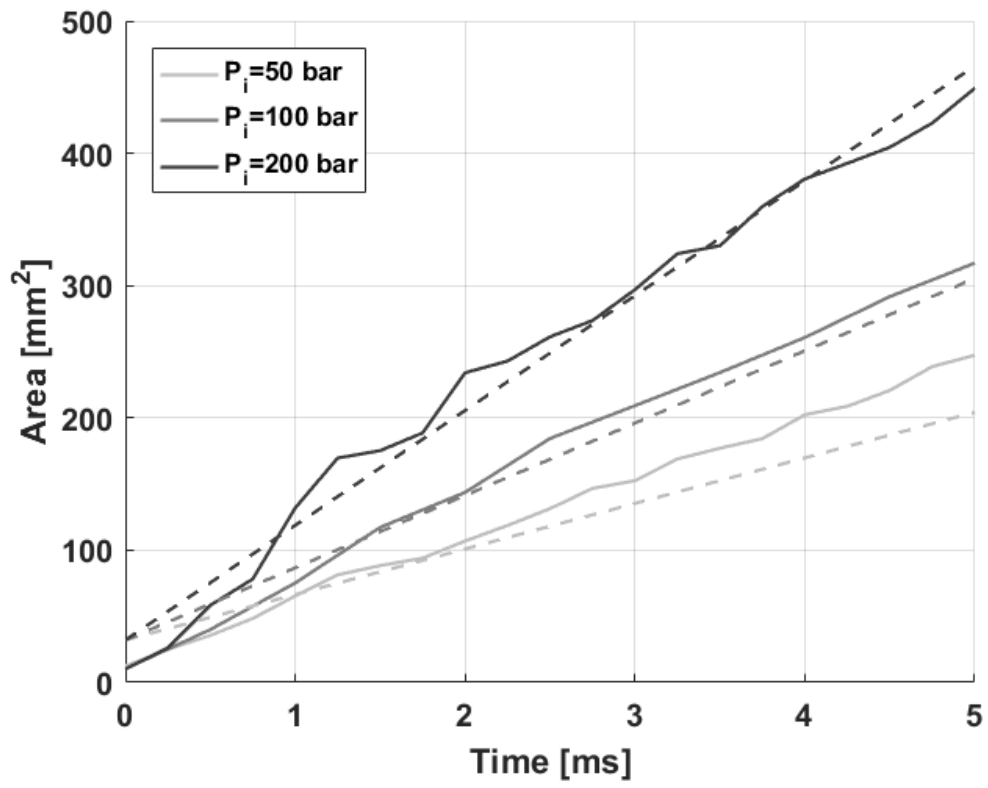

Figure 8. Wall film area time evolution for different fuel pressures $T_{i}=5 \mathrm{~ms}, d_{W}=50 \mathrm{~mm}$.

Figure 8 shows the film area time evolution for different fuel pressures. As expected, area is growing linearly with time. From one fuel pressure to another, the slope of the fitting curve is changing. It is admitted that $P_{d}$ varies linearly with $P_{i}$, while $Q$ varies as $\sqrt{P_{i}}$.

Based on the value of $K$ calculated above with $P_{i}=100 \mathrm{bar}$, one gets for the coefficient in Eq 10, for a fuel pressure $P_{1}$ :

$$
\left(\frac{P_{d} Q^{2}}{\pi^{2} \eta}\right)_{P_{1}}=\left(\frac{P_{1}}{10010^{5}}\right)^{2 / 3}\left(\frac{P_{d} Q^{2}}{\pi^{2} \eta}\right)_{100 \mathrm{bar}} .
$$

Eq 14 then enables to determine the slope of the fitting lines and gives 34.4 and 86.8 for $P_{i}=50$ bar and $P_{i}=200$ bar, respectively they are represented on Figure 8. It is clear that the dependency $R \propto P_{i}^{1 / 3}$ is correctly grasped by the model, however the prediction for the tests at $50 \mathrm{bar}$ is a bit bellow the experimental data. It could come from the value of $K_{m}$. Indeed it has been shown [14] that an increase in pressure causes a decrease in the deposited mass.

Eventually, the comparison between the model, and the experiment can be visualised on Figure 9. Here all the data collected at various pressure, injection duration and wall distance are displayed. The first bisector is represented in plain line to help visualise the quality of the model. Thus, Figure 9 shows the model fits well with the experimental measurements.

\section{Conclusions and perspectives}

The model presented in this paper is based on mass conservation and a steady-state balance between the spray pressure and viscous stresses. The model predictions are compared with experimental results obtained using the refractive index matching method and high-speed video imaging. Despite its simplicity, it clearly shows its ability to fairly predict the time evolution of the film radius $\left(R \propto t^{1 / 2}\right)$, as well as the influence of the fuel injection pressure $\left(R \propto P_{i}^{1 / 3}\right)$. It is also able to predict a wall film thickness with reasonable accuracy $\left(e \propto\left(\eta Q / P_{D}\right)^{1 / 3}\right)$.

The model is currently extended to take into account oblique spray-wall impingement, so as to be more representative of typical impact angles in GDI engines. Heat transfers and phase changes will be then added to include the effects of the strong vaporisation when the wall temperature reaches boiling conditions. Variation of fuels and greater pressure range will be done, in order to validate the evolution of $e$ as $P^{-1 / 6}$. 


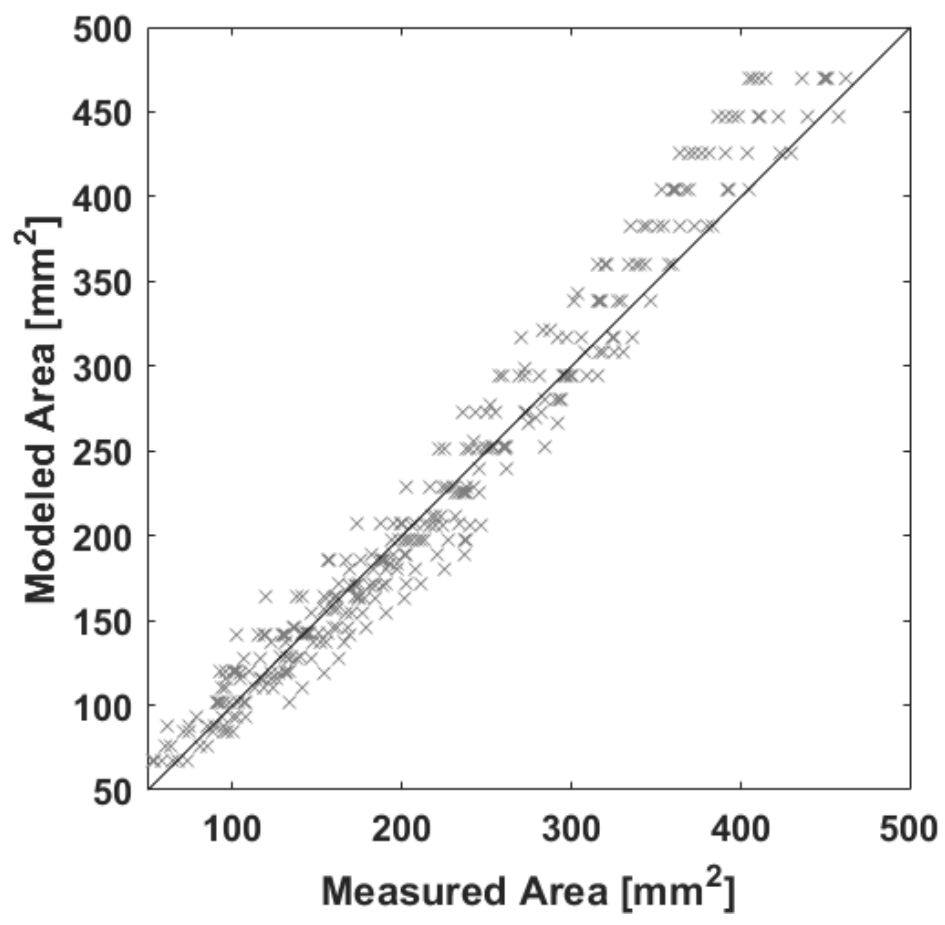

Figure 9. Comparison between measured and modeled area realised at 50, 100 and 200bar with injection time of 5.0ms and variable distance of injection

\section{Acknowledgements}

ANR and ANRT are acknowledged for their financial support.

\section{Nomenclature}

$V \quad$ Depth averaged radial velocity $\left[\mathrm{m} \cdot \mathrm{s}^{-1}\right]$

$v_{r} \quad$ Velocity in the fuel $\left[\mathrm{m} . \mathrm{s}^{-1}\right]$

$e \quad$ Thickness of the liquid film [m]

$P_{d} \quad$ Pressure transmitted by the spray $[\mathrm{Pa}]$

$Q \quad$ Discharge of the injector $\left[\mathrm{m}^{3} . \mathrm{s}^{-1}\right]$

$K_{P} \quad$ Pressure transmission coefficent

$K_{m} \quad$ Deposited mass ration

$K \quad$ Prefactor

$\eta \quad$ Dynamic viscosity [Pa.s]

$R \quad$ Radius of the wall film [m]

$A \quad$ Area of the wall film $\left[\mathrm{m}^{2}\right]$

$A_{s} \quad$ Area of spray impingement $\left[\mathrm{m}^{2}\right]$

$A_{o} \quad$ Area of the injector hole $\left[\mathrm{m}^{2}\right]$

$P_{i} \quad$ Pressure of injection $[\mathrm{Pa}]$

$T_{i} \quad$ Duration of injection [s]

$d_{W} \quad$ Injector-wall distance [m]

\section{References}

[1] Michael C Drake, Todd D Fansler, Arun S Solomon, and GA Szekely. Piston fuel films as a source of smoke and hydrocarbon emissions from a wall-controlled spark-ignited direct-injection engine. Technical report, SAE Technical Paper, 2003.

[2] Florian Steimle, Andre Kulzer, Herwig Richter, Dietmar Schwarzenthal, and Claudia Romberg. Systematic analysis and particle emission reduction of homogeneous direct injection si engines. Technical report, SAE Technical Paper, 2013.

[3] Nasser Ashgriz. Handbook of atomization and sprays: theory and applications. Springer Science \& Business Media, 2011.

[4] AL Yarin. Drop impact dynamics: splashing, spreading, receding, bouncing... Annu. Rev. Fluid Mech., 38:159-192, 2006.

[5] ALN Moreira, AS Moita, and MR Panao. Advances and challenges in explaining fuel spray impingement: How 
much of single droplet impact research is useful? Progress in energy and combustion science, 36(5):554-580, 2010.

[6] Sander Wildeman, Claas Willem Visser, Chao Sun, and Detlef Lohse. On the spreading of impacting drops. Journal of Fluid Mechanics, 805:636-655, 2016.

[7] Donald W Stanton and Christopher J Rutland. Modeling fuel film formation and wall interaction in diesel engines. Technical report, SAE Technical Paper, 1996.

[8] P.J. O'Rourke and A.A. Amsden. A spray/wall interaction submodel for the kiva-3 wall film model. Technical report, SAE Technical Paper, 2000.

[9] CX Bai, H Rusche, and AD Gosman. Modeling of gasoline spray impingement. Atomization and Sprays, 12(1-3), 2002.

[10] Davood Kalantari and Cameron Tropea. Spray impact onto flat and rigid walls: Empirical characterization and modelling. International Journal of Multiphase Flow, 33(5):525-544, 2007.

[11] Bo Yang and Jaal Ghandhi. Measurement of diesel spray impingement and fuel film characteristics using refractive index matching method. Technical report, SAE Technical Paper, 2007.

[12] David Maligne and Gilles Bruneaux. Time-resolved fuel film thickness measurement for direct injection si engines using refractive index matching. Technical report, SAE Technical Paper, 2011.

[13] Yangbing Zeng and Chia-Fon Lee. Multicomponent-fuel film-vaporization model for multidimensional computations. Journal of Propulsion and Power, 16(6):964-973, 2000.

[14] F Schulz, W Samenfink, J Schmidt, and F Beyrau. Systematic lif fuel wall film investigation. Fuel, 172:284292, 2016.

[15] Kyungnam Ko and Masataka Arai. Diesel spray impinging on a flat wall, part i: Characteristics of adhered fuel film in an impingement diesel spray. Atomization and Sprays, 12(5\&6), 2002.

[16] C Weiss. The liquid deposition fraction of sprays impinging vertical walls and flowing films. International journal of multiphase flow, 31(1):115-140, 2005.

The Maximum length of an ILASS 2017 paper should be 8 pages including all sections of the paper (abstract, keywords, introduction, main body, conclusions, acknowledgement, nomenclature and references. Your manuscript must be submitted in PDF file format through the ILASS 2017 webpage. The maximum file size must not exceed 10 MB. (regular text will be Arial 9 with 1.15 line spacing) 